Introduction to Corrosion Science 
E. McCafferty

\section{Introduction to Corrosion Science}

照 Springer 
E. McCafferty

Alexandria VA 22309

USA

ISBN 978-1-4419-0454-6

e-ISBN 978-1-4419-0455-3

DOI 10.1007/978-1-4419-0455-3

Springer New York Dordrecht Heidelberg London

Library of Congress Control Number: 2009940577

C Springer Science+Business Media, LLC 2010

All rights reserved. This work may not be translated or copied in whole or in part without the written permission of the publisher (Springer Science+Business Media, LLC, 233 Spring Street, New York, NY 10013, USA), except for brief excerpts in connection with reviews or scholarly analysis. Use in connection with any form of information storage and retrieval, electronic adaptation, computer software, or by similar or dissimilar methodology now known or hereafter developed is forbidden.

The use in this publication of trade names, trademarks, service marks, and similar terms, even if they are not identified as such, is not to be taken as an expression of opinion as to whether or not they are subject to proprietary rights.

Printed on acid-free paper

Springer is part of Springer Science+Business Media (www.springer.com) 


\section{Preface}

This textbook is intended for a one-semester course in corrosion science at the graduate or advanced undergraduate level. The approach is that of a physical chemist or materials scientist, and the text is geared toward students of chemistry, materials science, and engineering. This textbook should also be useful to practicing corrosion engineers or materials engineers who wish to enhance their understanding of the fundamental principles of corrosion science.

It is assumed that the student or reader does not have a background in electrochemistry. However, the student or reader should have taken at least an undergraduate course in materials science or physical chemistry. More material is presented in the textbook than can be covered in a one-semester course, so the book is intended for both the classroom and as a source book for further use.

This book grew out of classroom lectures which the author presented between 1982 and the present while a professorial lecturer at George Washington University, Washington, DC, where he organized and taught a graduate course on "Environmental Effects on Materials." Additional material has been provided by over 30 years of experience in corrosion research, largely at the Naval Research Laboratory, Washington, DC and also at the Bethlehem Steel Company, Bethlehem, PA and as a Robert A. Welch Postdoctoral Fellow at the University of Texas.

The text emphasizes basic principles of corrosion science which underpin extensions to practice. The emphasis here is on corrosion in aqueous environments, although a chapter on high-temperature oxidation has also been included. The overall effort has been to provide a brief but rigorous introduction to corrosion science without getting mired in extensive individual case histories, specific engineering applications, or compilations of practical corrosion data. Some other possible topics of interest in the field of corrosion science have not been included in accordance with the goal to keep the material introductory in nature and to keep the size of the book manageable. In addition, references are meant to be illustrative rather than exhaustive.

Most chapters also contain a set of problems. Numerical answers to problems are found at the end of the book.

Finally, the author wishes to recognize the various mentors who have graciously shaped his professional life. These are: Dr. J. B. Horton and A. R. Borzillo of the Bethlehem Steel Corporation, who introduced the author to the field of corrosion; the late Prof. A. C. Zettlemoyer of Lehigh University, who taught the author the beauty of surface chemistry while his $\mathrm{Ph}$. D. advisor; the late Dr. Norman Hackerman, postdoctoral mentor at the University of Texas; the late Dr. B. F. Brown and M. H. Peterson of the Naval Research Laboratory; and Prof. James P. Wightman of the Virginia Polytechnic Institute and State University, a "surface agent extra-ordinaire" with whom the author has spent an enjoyable and exciting sabbatical year.

The author is also grateful to Harry N. Jones, III, James R. Martin, Farrel J. Martin, Paul M. Natishan, Virginia DeGeorgi, Luke Davis, Robert A. Bayles, and Roy Rayne, all of the Naval Research Laboratory, who helped in various ways. The author also appreciates the kind assistance of 
A. Pourbaix of CEBELCOR (Centre Belge d'Etude de la Corrosion), C. Anderson Engh, Jr., M.D. of the Anderson Orthopaedic Clinic, Alexandria, VA; Phoebe Dent Weil, Northern Light Studio, Florence, MA; Erik Axdahl, and Harry's U-Pull-It, West Hazleton, PA.

Finally, the author wishes to thank Dr. Kenneth Howell, Senior Chemistry Editor at Springer, for his encouragement and support.

Washington, DC

E. McCafferty

2009 


\section{Contents}

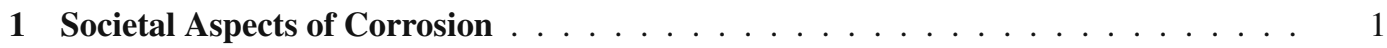

We Live in a Metals-Based Society . . . . . . . . . . . . . . . . . . . . . . . . . . 1

Why Study Corrosion? . . . . . . . . . . . . . . . . . . . . . . . 1

Corrosion and Human Life and Safety . . . . . . . . . . . . . . . . 1

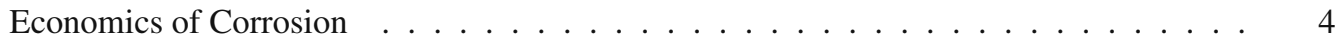

Corrosion and the Conservation of Materials . . . . . . . . . . . . . . . 5

The Study of Corrosion . . . . . . . . . . . . . . . . . . . . . 6

Corrosion Science vs. Corrosion Engineering . . . . . . . . . . . . . . . . . 8

Challenges for Today's Corrosion Scientist . . . . . . . . . . . . . . . . . . . . 9

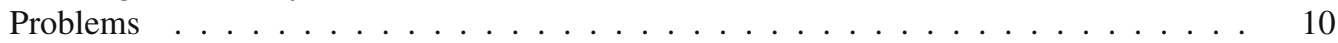

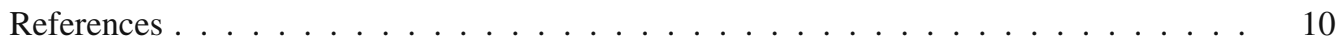

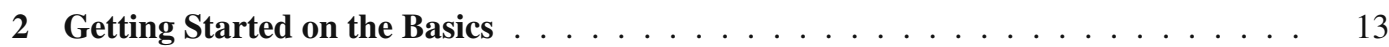

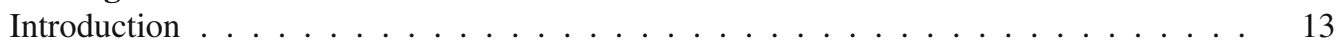

What is Corrosion? . . . . . . . . . . . . . . . . 13

Physical Processes of Degradation . . . . . . . . . . . . . . . . . . . 13

Environmentally Assisted Degradation Processes . . . . . . . . . . . . . . . 14

Electrochemical Reactions . . . . . . . . . . . . . . . . . . . . . . . . . . . . . . . . . . . .

Half-Cell Reactions . . . . . . . . . . . . . . . . . . . . . . . . . . . . . . . . . . . . . . . .

Anodic Reactions . . . . . . . . . . . . . . . . . . . . . . . 15

Cathodic Reactions . . . . . . . . . . . . . . . . . . . 16

Coupled Electrochemical Reactions . . . . . . . . . . . . . . . . . . . . 17

A Note About Atmospheric Corrosion . . . . . . . . . . . . . . . . . . . . . . 18

Secondary Effects of Cathodic Reactions . . . . . . . . . . . . . . . . . . . 19

Three Simple Properties of Solutions . . . . . . . . . . . . . . . . . . . . . . 21

The Faraday and Faraday's Law . . . . . . . . . . . . . . . . . . . . . . . . . . . . . . . . . . . . . . . . . . .

Units for Corrosion Rates . . . . . . . . . . . . . . . . . . . . . . . . . . . . . . . . . . . . . . . . . . . . . . . . .

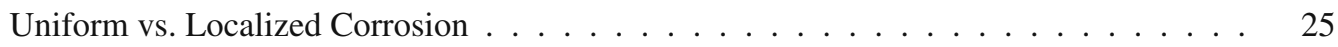

The Eight Forms of Corrosion . . . . . . . . . . . . . . . 27

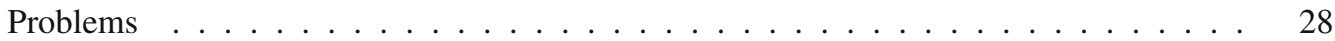

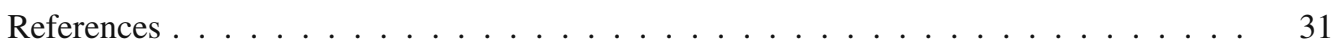

3 Charged Interfaces . . . . . . . . . . . . . . . . . . . . 33

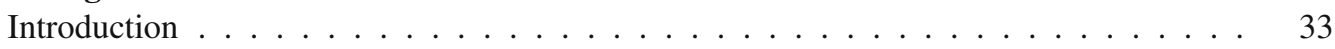

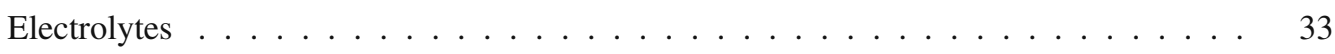

The Interior of an Electrolyte . . . . . . . . . . . . . . . . . 33

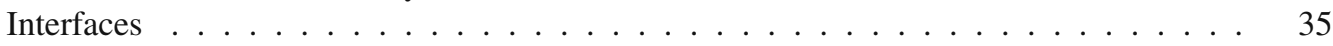


Encountering an Interface $\ldots \ldots \ldots \ldots \ldots \ldots \ldots$

The Solution/Air Interface . . . . . . . . . . . . . . . . . . . . . 36

The Metal/Solution Interface . . . . . . . . . . . . . . . . . . . . 37

Metal Ions in Two Different Chemical Environments . . . . . . . . . . . . . . . . 38

The Electrical Double Layer . . . . . . . . . . . . . . . . . . . . . . . . . . . . . . . . . . . . . . . .

The Gouy-Chapman Model of the Electrical Double Layer . . . . . . . . . . . . . . . 40

The Electrostatic Potential and Potential Difference . . . . . . . . . . . . . . . . 40

The Stern Model of the Electrical Double Layer . . . . . . . . . . . . . . . . . . . . . 41

The Bockris-Devanathan-Müller Model of the Electrical Double Layer . . . . . . . 42

Significance of the Electrical Double Layer to Corrosion . . . . . . . . . . . . . . . . 43

Electrode Potentials . . . . . . . . . . . . . . . . . . . . . . 4 44

The Potential Difference Across a Metal/Solution Interface . . . . . . . . . . . . . . . . . 44

Relative Electrode Potentials . . . . . . . . . . . . . . . . . . . . . . . . . . . . . . . . . 45

The Electromotive Force Series . . . . . . . . . . . . . . . . . . . 46

Reference Electrodes for the Laboratory and the Field . . . . . . . . . . . . . . . . . 48

Measurement of Electrode Potentials . . . . . . . . . . . . . . . . . . . 52

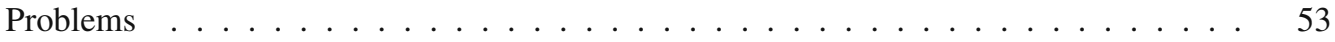

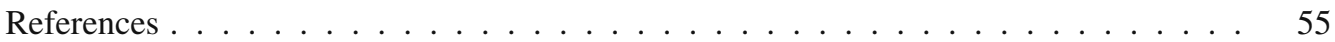

4 A Brief Review of Thermodynamics . . . . . . . . . . . . . . . . . . 57

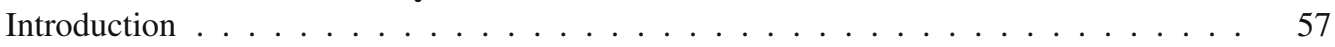

Thermodynamic State Functions . . . . . . . . . . . . . . . . 57

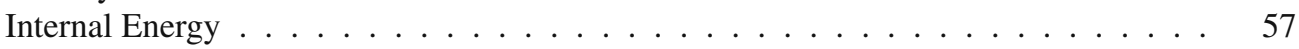

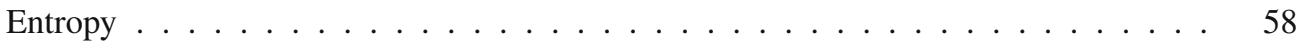

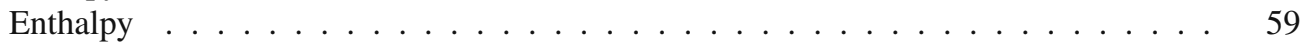

Helmholtz and Gibbs Free Energies . . . . . . . . . . . . . . . . . . 59

Free Energy and Spontaneity . . . . . . . . . . . . . . . . . . . 60

Relationships Between Thermodynamic Functions . . . . . . . . . . . . 61

The Chemical Potential and Standard States . . . . . . . . . . . . . . . . . . . 63

More About the Chemical Potential . . . . . . . . . . . . . . . . . . 63

A Note About Units for $\Delta G^{o}$ or $\Delta G \ldots \ldots \ldots \ldots \ldots \ldots \ldots$

The Free Energy and Electrode Potentials . . . . . . . . . . . . . . . . 65

The Nernst Equation . . . . . . . . . . . . . . . . . . . . . . . 66

Standard Free Energy Change and the Equilibrium Constant . . . . . . . . . . 67

A Quandary - The Sign of Electrode Potentials . . . . . . . . . . . . . . . . . . 68

Factors Affecting Electrode Potentials . . . . . . . . . . . . . . . . . . . . . 69

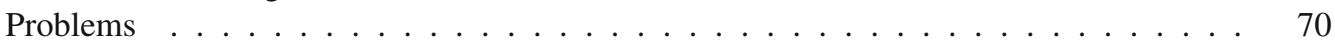

References . . . . . . . . . . . . . . . . . 72

5 Thermodynamics of Corrosion: Electrochemical Cells

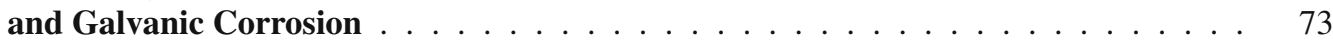

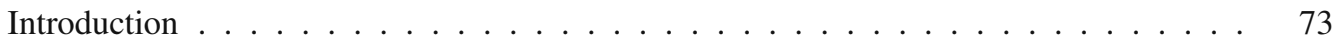

Electrochemical Cells . . . . . . . . . . . . . . . . . . . . 73

Electrochemical Cells on the Same Surface . . . . . . . . . . . . . 75

Galvanic Corrosion . . . . . . . . . . . . . . . . . . 76

Galvanic Series . . . . . . . . . . . . . . . . . . . . . . . . . 76

Cathodic Protection . . . . . . . . . . . . . . . . . . . . . 79

Two Types of Metallic Coatings . . . . . . . . . . . . . . . . . . . . 80

Titanium Coatings on Steel: A Research Study . . . . . . . . . . . . . . . 82 
Protection Against Galvanic Corrosion . . . . . . . . . . . . . . . . . . . 83

Differential Concentration Cells . . . . . . . . . . . . . . . . . . . . 84

Metal Ion Concentration Cells . . . . . . . . . . . . . . . . . . . 84

Oxygen Concentration Cells . . . . . . . . . . . . . . . . . . . . 86

The Evans Water Drop Experiment . . . . . . . . . . . . . . . . . . . . . 88

Waterline Corrosion . . . . . . . . . . . . . . . . . . 88

Crevice Corrosion: A Preview . . . . . . . . . . . . . . . . . . . . . 89

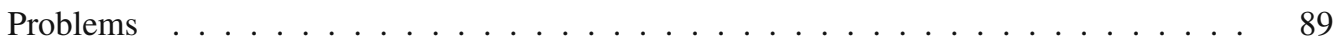

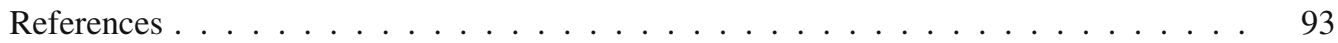

6 Thermodynamics of Corrosion: Pourbaix Diagrams . . . . . . . . . . . . . . . 95

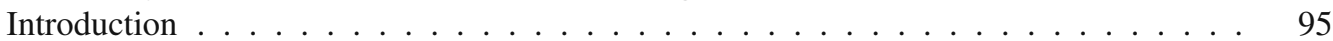

Pourbaix Diagram for Aluminum . . . . . . . . . . . . . . . . . . . . 96

Construction of the Pourbaix Diagram for Aluminum . . . . . . . . . . . . . . . 96

Comparison of Thermodynamic and Kinetic Data for Aluminum . . . . . . . . . . . . 101

Pourbaix Diagram for Water . . . . . . . . . . . . . . . . . . . . . . . . . 101

Pourbaix Diagrams for Other Metals . . . . . . . . . . . . . . . . . . . . . . 103

Pourbaix Diagram for Zinc . . . . . . . . . . . . . . . . . . 103

Pourbaix Diagram for Iron . . . . . . . . . . . . . . . . . . . . 103

Pourbaix Diagrams for Additional Metals . . . . . . . . . . . . . . . . . . . . . 106

Applications of Pourbaix Diagrams to Corrosion . . . . . . . . . . . . . . . . . . . 108

Limitations of Pourbaix Diagrams . . . . . . . . . . . . . . . . . . 111

Pourbaix Diagrams for Alloys . . . . . . . . . . . . . . . . . . 111

Pourbaix Diagrams at Elevated Temperatures . . . . . . . . . . . . . . . . 112

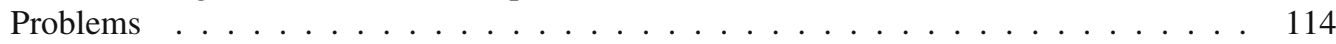

References . . . . . . . . . . . . . . . . . . . . 116

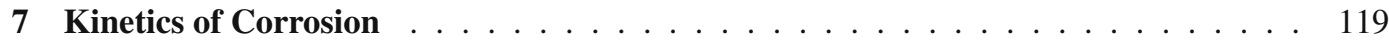

Introduction . . . . . . . . . . . . . . . . . . . . . . . . . 119

Units for Corrosion Rates . . . . . . . . . . . . . . . . . . . . . . . 119

Methods of Determining Corrosion Rates . . . . . . . . . . . . . . . . . . . . . . . . . . . . . . . . . . 12

Weight Loss Method . . . . . . . . . . . . . . . . . . . . . . 120

Weight Gain Method . . . . . . . . . . . . . . . . . . . . 120

Chemical Analysis of Solution . . . . . . . . . . . . . . . . . . . . . 121

Gasometric Techniques . . . . . . . . . . . . . . . . . . . . 122

Thickness Measurements . . . . . . . . . . . . . . . . . . . . . . . . . 124

Electrical Resistance Method . . . . . . . . . . . . . . . . . . . . . . . . . . . 124

Inert Marker Method . . . . . . . . . . . . . . . . . . . . . . . . . . . . . . . . . . . . . . . . . . . . . . . .

Electrochemical Techniques . . . . . . . . . . . . . . . . . . . . . 126

Electrochemical Polarization . . . . . . . . . . . . . . . . . . . 127

Anodic and Cathodic Polarization . . . . . . . . . . . . . . . . . . . . 127

Visualization of Cathodic Polarization . . . . . . . . . . . . . . . . . . . 127

Visualization of Anodic Polarization . . . . . . . . . . . . . . . . . 128

Ohmic Polarization . . . . . . . . . . . . . . . . . . . 130

Electrode Kinetics for Activation Polarization . . . . . . . . . . . . . . . . . . 131

Absolute Reaction Rate Theory . . . . . . . . . . . . . . . . . . . . . . . 131

Electrode Kinetics for Non-Corroding Metals . . . . . . . . . . . . . . . . . . . . . . . . . . . . . 132

How to Plot Polarization Curves? . . . . . . . . . . . . . . . . . . . . 136

The Tafel Equation . . . . . . . . . . . . . . . . . . . . . . 138 
Reversible and Irreversible Potentials . . . . . . . . . . . . . . . . . . . . . . . 139

Mixed Potential Theory (Wagner and Traud) . . . . . . . . . . . . . . . . . . . 140

Electrode Kinetic Parameters . . . . . . . . . . . . . . . . . . . . . . . . . . . . . . 144

Applications of Mixed Potential Theory . . . . . . . . . . . . . . . . . . . . . . 146

Metals in Acid Solutions . . . . . . . . . . . . . . . . . . . . . . . . . . . . . . . . . . . . . . . . . . . . . . . . . . .

Tafel Extrapolation . . . . . . . . . . . . . . . . . . . . . . . . . 148

Verification of Corrosion Rates Obtained by Tafel Extrapolation . . . . . . . . . . 150

Cathodic Protection of Iron in Acids . . . . . . . . . . . . . . . . . . 150

Effect of the Cathodic Reaction . . . . . . . . . . . . . . . . . . . 154

Effect of Cathode Area on Galvanic Corrosion . . . . . . . . . . . . . . . . . 154

Multiple Oxidation-Reduction Reactions . . . . . . . . . . . . . . . . . 156

Anodic or Cathodic Control . . . . . . . . . . . . . . . . . . . . . . 158

The Linear Polarization Method (Stern and Geary) . . . . . . . . . . . . . . . . . . 159

Advantages and Possible Errors for the Linear Polarization Technique . . . . . . . . . . 162

Applications of the Linear Polarization Technique . . . . . . . . . . . . . . 163

Small-Amplitude Cyclic Voltammetry . . . . . . . . . . . . . . . . . . . . . . . 164

Experimental Techniques for Determination of Polarization Curves . . . . . . . . . . . 165

Electrode Samples . . . . . . . . . . . . . . . . . . . . . 165

Electrode Holders . . . . . . . . . . . . . . . . . . . . . . . . . . . . . . . 166

Electrochemical Cells . . . . . . . . . . . . . . . . . . . . 167

Instrumentation and Procedures . . . . . . . . . . . . . . . . . . 168

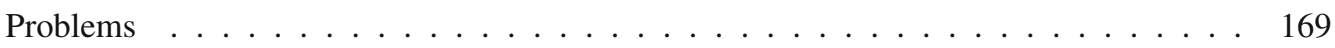

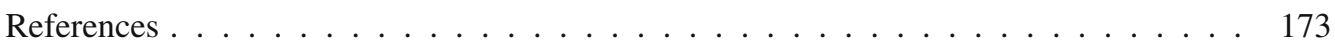

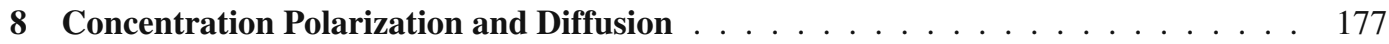

Introduction . . . . . . . . . . . . . . . . . . . . . . . 177

Where Oxygen Reduction Occurs ． . . . . . . . . . . . . . . . . . . . 177

Concentration Polarization in Current Density-Potential Plots . . . . . . . . . . . . . 178

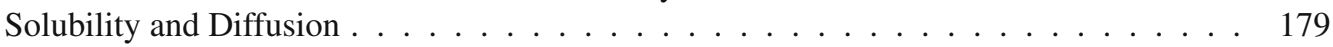

Solubility of Oxygen in Aqueous Solutions . . . . . . . . . . . . . . . . . . . 179

Fick's First Law of Diffusion . . . . . . . . . . . . . . . . . . . . . . . . . 181

Diffusion and Random Walks . . . . . . . . . . . . . . . . . . . . . 183

Electrode Kinetics for Concentration Polarization . . . . . . . . . . . . . . . . . 186

Concentration Profile Near an Electrode Surface . . . . . . . . . . . . . . . . . 186

Limiting Diffusion Current Density . . . . . . . . . . . . . . . . . . . . . 187

Diffusion Layer vs. The Diffuse Layer . . . . . . . . . . . . . . . . . . . . . . 189

Current-Potential Relationship for Concentration Polarization . . . . . . . . . . . . . 189

Wagner-Traud Theory for Concentration Polarization . . . . . . . . . . . . . . . 191

Effect of Environmental Factors on Concentration Polarization and Corrosion . . . . . . 192

Effect of Oxygen Concentration . . . . . . . . . . . . . . . . . . . 193

Effect of Solution Velocity . . . . . . . . . . . . . . . . . . . 194

Effect of Temperature . . . . . . . . . . . . . . . . . . . . 196

Further Applications of Concentration Polarization Curves . . . . . . . . . . . . . . . . 197

Cathodic Protection . . . . . . . . . . . . . . . . . . . . . . . . . 197

Area Effects in Galvanic Corrosion . . . . . . . . . . . . . . . . . . . . . 199

Linear Polarization . . . . . . . . . . . . . . . . . . . . . . . . . 199

Concentration Polarization in Acid Solutions . . . . . . . . . . . . . . . . . . 200

Combined Activation and Concentration Polarization . . . . . . . . . . . . . 202 
The Rotating Disc Electrode . . . . . . . . . . . . . . . . . . . . . . . 202

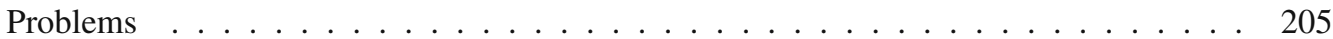

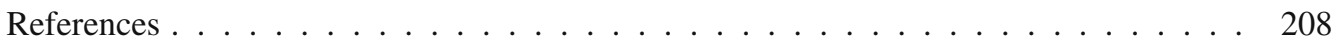

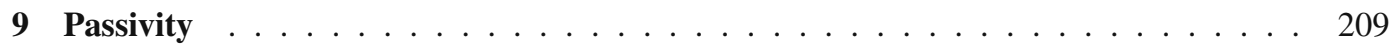

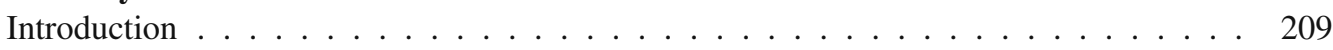

Aluminum: An Example . . . . . . . . . . . . . . . . . . . . . 209

What is Passivity? . . . . . . . . . . . . . . . . . . . 210

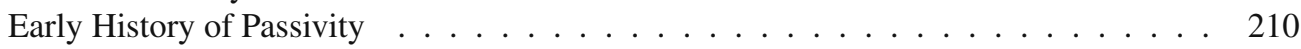

Thickness of Passive Oxide Films . . . . . . . . . . . . . . . . . . . . 210

Purpose of This Chapter . . . . . . . . . . . . . . . . . . . . 211

Electrochemical Basis for Passivity . . . . . . . . . . . . . . . . . . . . 211

Theories of Passivity . . . . . . . . . . . . . . . . . . . . . . . 215

Adsorption Theory . . . . . . . . . . . . . . . . . . . . 215

Oxide Film Theory . . . . . . . . . . . . . . . . . . . . . . . . . . . 216

Film Sequence Theory . . . . . . . . . . . . . . . . . . . . . . . 218

Surface Analysis Techniques for the Examination of Passive Films . . . . . . . . . . . . 218

X-ray Photoelectron Spectroscopy (XPS) ～. . . . . . . . . . . . . . . 220

X-ray Absorption Spectroscopy ． . . . . . . . . . . . . . . . . . . . . 222

Scanning Tunneling Microscopy . . . . . . . . . . . . . . . . . . . . . . 223

Models for the Passive Oxide Film on Iron . . . . . . . . . . . . . . . . . . . . . . . . . . . . . . . . . . . . . . 224

Bilayer Model . . . . . . . . . . . . . . . . . . . . . . . . . . . . . . . . . . . . . . . . . . . . 224

Hydrous Oxide Model . . . . . . . . . . . . . . . . . . . . . . . . 227

Bipolar-Fixed Charge Model . . . . . . . . . . . . . . . . . . . . . 228

Spinel/Defect Model . . . . . . . . . . . . . . . . . . . . . . . . . . . . . . . . 229

What Do These Various Models Mean? . . . . . . . . . . . . . . . . . . . . . 230

Passive Oxide Films on Aluminum . . . . . . . . . . . . . . . . . . . . . . . . 230

Air-Formed Oxide Films . . . . . . . . . . . . . . . . . . . . . . . 231

Films Formed in Aqueous Solutions . . . . . . . . . . . . . . . . . . . 231

Properties of Passive Oxide Films . . . . . . . . . . . . . . . . . . . . . . . 232

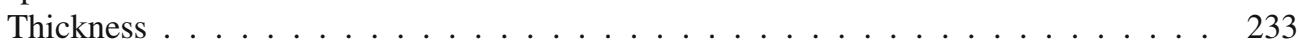

Electronic and Ionic Conductivity . . . . . . . . . . . . . . . . . . 233

Chemical Stability . . . . . . . . . . . . . . . . . . 233

Mechanical Properties . . . . . . . . . . . . . . . . . . . . 234

Structure of Passive Films . . . . . . . . . . . . . . . . . . . . . 235

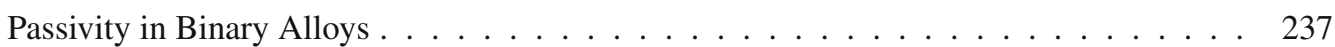

Electron Configuration Theory . . . . . . . . . . . . . . . . . . . . . . . . . . . . . . . . . . . . . 238

Oxide Film Properties . . . . . . . . . . . . . . . . . . . . . . . . 241

Percolation Theory . . . . . . . . . . . . . . . . . . . . . . . . . . . . . . . . . . . . . . . . . . 242

Graph Theory Model . . . . . . . . . . . . . . . . . . . . . . . . . 243

Passivity in Stainless Steels . . . . . . . . . . . . . . . . . . . . . . . . . . 249

Electrochemical Aspects . . . . . . . . . . . . . . . . . . . 250

Composition of Passive Films on Stainless Steels . . . . . . . . . . . . . . . . 252

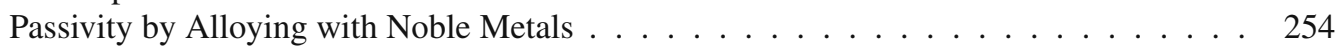

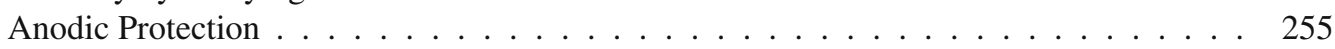

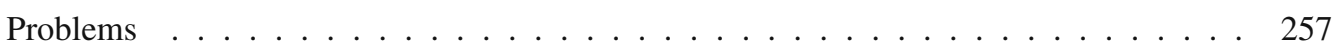

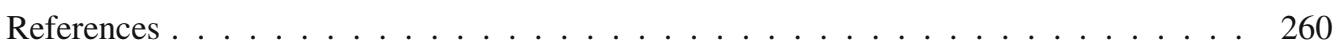




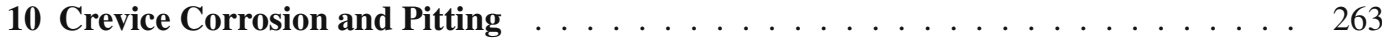

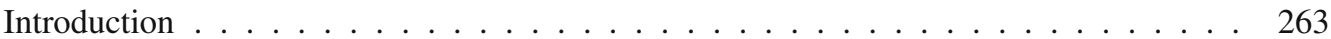

Crevice Corrosion . . . . . . . . . . . . . . . . . . . . . . 263

Initiation of Crevice Corrosion . . . . . . . . . . . . . . . . . . . 264

Propagation of Crevice Corrosion . . . . . . . . . . . . . . . 269

Crevice Corrosion Testing . . . . . . . . . . . . . . . . . . 272

Area Effects in Crevice Corrosion . . . . . . . . . . . . . . . . . . . . 274

Protection Against Crevice Corrosion . . . . . . . . . . . . . . . . . 275

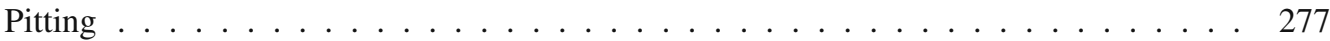

Critical Pitting Potential . . . . . . . . . . . . . . . . . . 278

Experimental Determination of Pitting Potentials . . . . . . . . . . . . . . . 280

Effect of Chloride Ions on the Pitting Potential . . . . . . . . . . . . . . . . . 282

Effect of Inhibitors on the Pitting Potential _ . . . . . . . . . . . . . . . . . . 283

Mechanism of Pit Initiation . . . . . . . . . . . . . . . . . . . 283

Mechanism of Pit Propagation . . . . . . . . . . . . . . . . 286

Protection Potential . . . . . . . . . . . . . . . . . . . . . 288

Metastable Pits and Repassivation . . . . . . . . . . . . . . . . . . . . . . 290

Experimental Pourbaix Diagrams for Pitting . . . . . . . . . . . . . . . . . . 291

Effect of Molybdenum on the Pitting of Stainless Steels . . . . . . . . . . . . . 293

Effect of Sulfide Inclusions on the Pitting of Stainless Steels . . . . . . . . . . . . . . 294

Effect of Temperature . . . . . . . . . . . . . . . . . . . . . . . . . . . . . . . . . . . . . . . . . 294

Protection Against Pitting . . . . . . . . . . . . . . . . . . . . . 296

Pitting of Aluminum . . . . . . . . . . . . . . . . . . . . 297

Occluded Corrosion Cells . . . . . . . . . . . . . . . . . . . . . 300

Occluded Corrosion Cell (OCC) on Iron . . . . . . . . . . . . . . . . . 301

Occluded Corrosion Cells on Copper and Aluminum . . . . . . . . . . . . . . . 303

Differences Between Pitting and Crevice Corrosion . . . . . . . . . . . . . . . . 306

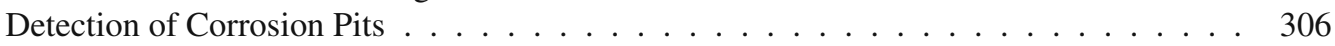

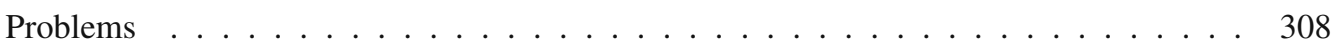

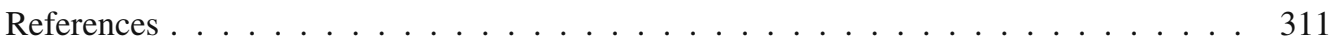

11 Mechanically Assisted Corrosion . . . . . . . . . . . . . . . . . . 315

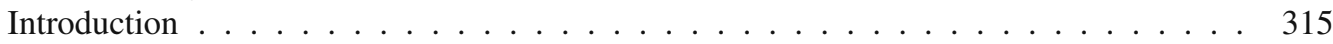

Stress-Corrosion Cracking $\ldots \ldots \ldots \ldots \ldots \ldots \ldots$

Mechanical Metallurgy . . . . . . . . . . . . . . . . . . . . . . 318

Characteristics of Stress-Corrosion Cracking . . . . . . . . . . . . . . . . 319

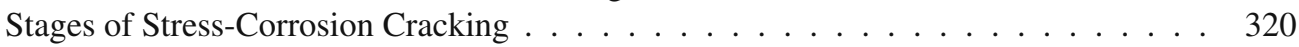

Fracture Mechanics and SCC . . . . . . . . . . . . . . . . . . . . . . . 323

SCC Testing . . . . . . . . . . . . . . . . . . . . . . . . 331

Interpretation of SCC Test Data . . . . . . . . . . . . . . . . . . 334

Metallurgical Effects in SCC . . . . . . . . . . . . . . . 335

Environmental Effects on SCC . . . . . . . . . . . . . . . . . . . 336

Mechanisms of SCC . . . . . . . . . . . . . . . . . . . . . . . 339

Protection Against Stress-Corrosion Cracking . . . . . . . . . . . . . . . . . 345

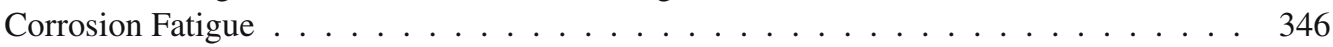

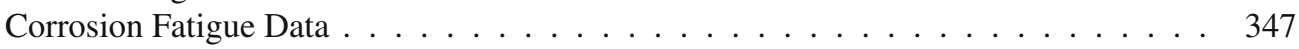

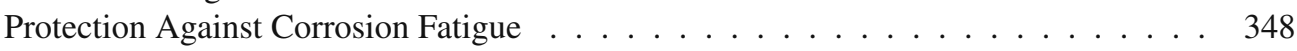

Cavitation Corrosion . . . . . . . . . . . . . . . . . . . . 349 
Erosion Corrosion and Fretting Corrosion _ . . . . . . . . . . . . . . . 352

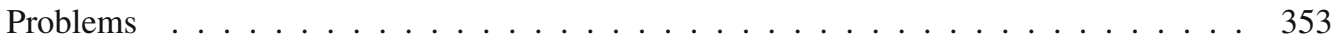

References . . . . . . . . . . . . . . . . . . . 354

12 Corrosion Inhibitors . . . . . . . . . . . . . . . . . . . . . . . 357

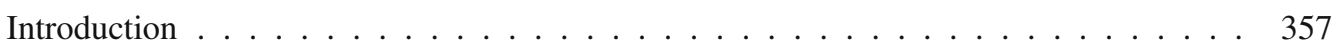

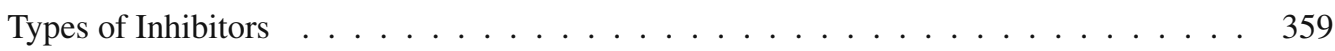

Acidic Solutions . . . . . . . . . . . . . . . . . . . . . . . 360

Chemisorption of Inhibitors $\ldots \ldots \ldots$. . . . . . . . . . . . . . 361

Effect of Inhibitor Concentration . . . . . . . . . . . . . . . . . 362

Chemical Factors in the Effectiveness of Chemisorbed Inhibitors . . . . . . . . . . . 363

Involvement of Water . . . . . . . . . . . . . . . . . . . . 367

Competitive vs. Co-operative Adsorption . . . . . . . . . . . . . . . . . . . 369

Effect of the Electrical Double Layer . . . . . . . . . . . . . . . . . . . . . . 370

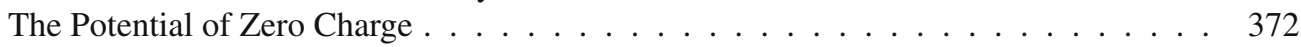

Effect of Molecular Structure . . . . . . . . . . . . . . . . 373

Adsorption Isotherms . . . . . . . . . . . . . . . . . . . . . 376

Nearly Neutral Solutions . . . . . . . . . . . . . . . . . . . . . . . . . 379

Effect of Oxide Films . . . . . . . . . . . . . . . . . . . . . . . . 379

Chelating Compounds as Corrosion Inhibitors . . . . . . . . . . . . . . . . . . . . . . . . . . . . . . . . . . . . . . .

Chromates and Chromate Replacements . . . . . . . . . . . . . . . . . . . . . 381

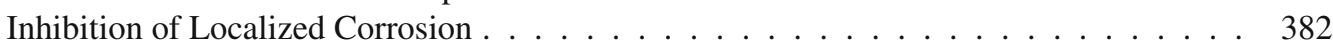

Pitting Corrosion . . . . . . . . . . . . . . . . . . . . . 382

Crevice Corrosion . . . . . . . . . . . . . . . . . . 386

Stress-Corrosion Cracking and Corrosion Fatigue . . . . . . . . . . . . . . . . . . . . 387

New Approaches to Corrosion Inhibition . . . . . . . . . . . . . . . . . . . . . . . . 389

Biological Molecules . . . . . . . . . . . . . . . . . . . . . . . . . . 390

Langmuir-Blodgett Films and Self-assembled Monolayers . . . . . . . . . . . . . . . 393

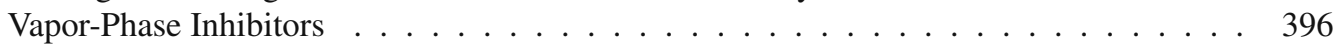

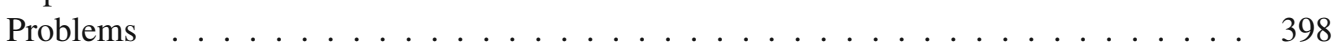

References ............................. 400

13 Corrosion Under Organic Coatings _ . . . . . . . . . . . . . . . . . . . . 403

Introduction . . . . . . . . . . . . . . . . . . . . 403

Paints and Organic Coatings . . . . . . . . . . . . . . . . . . . . 404

Underfilm Corrosion . . . . . . . . . . . . . . . . . . . . . 405

Water Permeation into an Organic Coating . . . . . . . . . . . . . . 406

Permeation of Oxygen and Ions into an Organic Coating . . . . . . . . . . . . . . 410

Breakdown of an Organic Coating . . . . . . . . . . . . . . . . . . . 411

Adhesion of Organic Coatings . . . . . . . . . . . . . . . . . . . . 412

Improved Corrosion Prevention by Coatings . . . . . . . . . . . . . . . . . . 416

Filiform Corrosion . . . . . . . . . . . . . . . . . . . . 417

Corrosion Tests for Organic Coatings . . . . . . . . . . . . . . . . . . . . . . . . . . 419

Accelerated Tests . . . . . . . . . . . . . . . . . . . . . . 419

Cathodic Delamination . . . . . . . . . . . . . . . . . . . . . . 419

AC Impedance Techniques - A Brief Comment . . . . . . . . . . . . . . . . . . . . . . 422

Recent Directions and New Challenges . . . . . . . . . . . . . . . . . . . . . 422

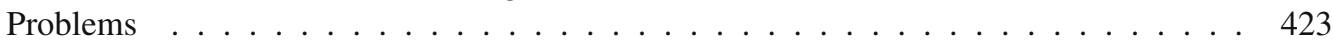

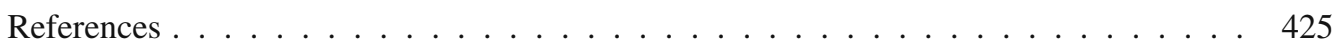


14 AC Impedance . . . . . . . . . . . . . . . . . . . . . . . . . . . . . . . . . . . . 427

Introduction . . . . . . . . . . . . . . . . . . . . . 427

Relaxation Processes . . . . . . . . . . . . . . . . . . . . . . . 427

Experimental Setup . . . . . . . . . . . . . . . . . . . . . . . . 429

Complex Numbers and AC Circuit Analysis . . . . . . . . . . . . . . . . . . . . 430

The Metal/Solution Interface . . . . . . . . . . . . . . . . . . . . . . . 431

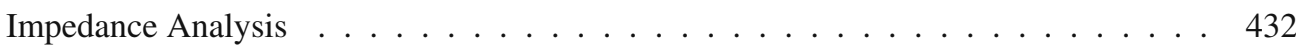

Additional Methods of Plotting Impedance Data . . . . . . . . . . . . . . . . . 434

Multiple Time Constants and the Effect of Diffusion . . . . . . . . . . . . . . . 436

Kramers-Kronig Transforms . . . . . . . . . . . . . . . . . . . . . . . . . 437

Application to Corrosion Inhibition . . . . . . . . . . . . . . . . 438

Organic Coatings . . . . . . . . . . . . . . . . . . . . . 441

Oxide Films and Surface Treatments . . . . . . . . . . . . . . . . . . . . . . . . . 446

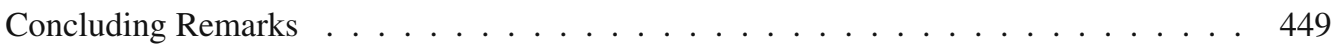

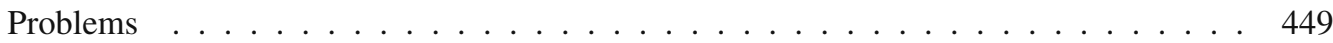

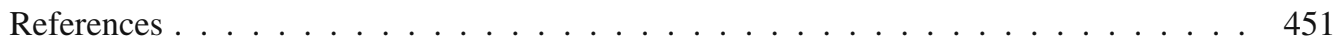

15 High-Temperature Gaseous Oxidation ～. . . . . . . . . . . . . . . . . . . 453

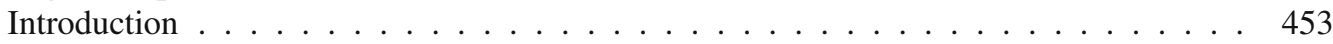

Thermodynamics of High-Temperature Oxidation . . . . . . . . . . . . . . . . . 453

Ellingham Diagrams . . . . . . . . . . . . . . . . . . . . . . . 454

Equilibrium Pressure of Oxygen . . . . . . . . . . . . . . . . . . . . . . 455

Theory of High-Temperature Oxidation . . . . . . . . . . . . . . . . . . . 456

Oxidation Rate Laws . . . . . . . . . . . . . . . . . . . . . . . . . . . . . . . . . . . . . . . . . . . . . . .

Linear Rate Law . . . . . . . . . . . . . . . . . . . . . . . . . 458

Parabolic Rate Law . . . . . . . . . . . . . . . . . . . . . . . . 459

Logarithmic Rate Law . . . . . . . . . . . . . . . . . . . . . . . . . 460

Comparison of Rate Laws . . . . . . . . . . . . . . . . . . . . . . . . 460

The Wagner Mechanism and the Parabolic Rate Law . . . . . . . . . . . . . . . . . . . 460

Effect of Temperature on the Oxidation Rate . . . . . . . . . . . . . . . . . . . . . 463

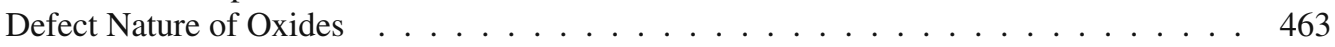

Semiconductor Nature of Oxides . . . . . . . . . . . . . . . . . . . 465

Hauffe Rules for Oxidation . . . . . . . . . . . . . . . . . . . . . . . . . 466

Effect of Oxygen Pressure on Parabolic Rate Constants . . . . . . . . . . . . . . . . . 471

Non-uniformity of Oxide Films . . . . . . . . . . . . . . . . . . . . . . . 472

Protective vs. Non-protective Oxides . . . . . . . . . . . . . . . . . . . . . . . . . . . . . . . . . . 473

Pilling-Bedworth Ratio . . . . . . . . . . . . . . . . . . . . . . 473

Properties of Protective High-Temperature Oxides . . . . . . . . . . . . . . . . . . 473

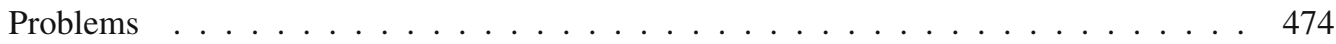

References . . . . . . . . . . . . . . . . . . 475

16 Selected Topics in Corrosion Science . . . . . . . . . . . . . . . . . . . . . . . 477

Introduction . . . . . . . . . . . . . . . . . . . . . . . 477

Electrode Kinetics of Iron Dissolution in Acids . . . . . . . . . . . . . . . . . 477

Bockris-Kelly Mechanism . . . . . . . . . . . . . . . . . . . . . . . . 478

Heusler Mechanism . . . . . . . . . . . . . . . . . . . . . . . . . 480

Reconciliation of the Two Mechanisms . . . . . . . . . . . . . . . . . . 481

Additional Work on Electrode Kinetics . . . . . . . . . . . . . . . . . . . . . . 482

Distribution of Current and Potential . . . . . . . . . . . . . . . 483 
Laplace's Equation . . . . . . . . . . . . . . . . . . . . . . . . . 483

Circular Corrosion Cells . . . . . . . . . . . . . . . . . . . . . . 483

Parametric Study . . . . . . . . . . . . . . . . . . . . . . . . 486

Application to the Experiments of Rozenfeld and Pavlutskaya . . . . . . . . . . . . . 488

Large Structures and Scaling Rules . . . . . . . . . . . . . . . . . . . . . . . . . . . . . . . . . . 499

Modeling of the Cathodic Protection System of a Ship . . . . . . . . . . . . . . . . 491

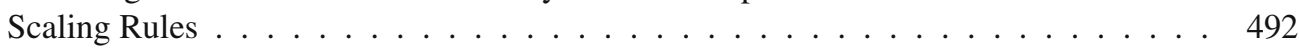

Acid-Base Properties of Oxide Films . . . . . . . . . . . . . . . . . . . . . . . . . . 494

Surface Hydroxyl Groups ． . . . . . . . . . . . . . . . . . . . . . . . . . . 494

Nature of Acidic and Basic Surface Sites . . . . . . . . . . . . . . . . . . 495

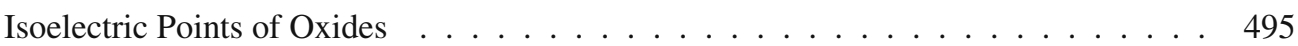

Surface Charge and Pitting . . . . . . . . . . . . . . . . . . . . . 497

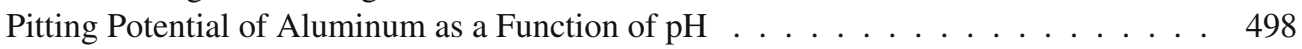

Surface Modification by Directed Energy Beams . . . . . . . . . . . . . . . . . . . . . 499

Ion Implantation and Related Processes . . . . . . . . . . . . . . . . . . . . . . . . . . . . . . . . . . . . . . . . . . . . .

Applications of Ion Implantation . . . . . . . . . . . . . . . . . . 501

Laser-Surface Processing Techniques . . . . . . . . . . . . . . . 505

Applications of Laser-Surface Processing . . . . . . . . . . . . . . . . . 507

Comparison of Ion Implantation and Laser-Surface Processing . . . . . . . . . . . . 509

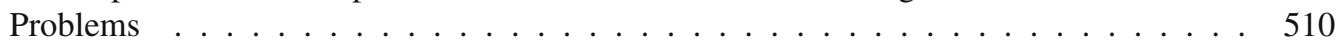

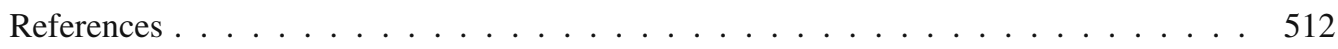

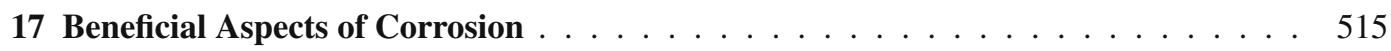

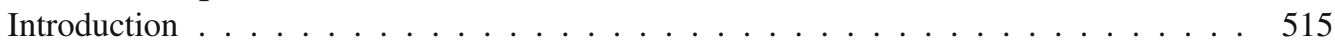

Rust Is Beautiful . . . . . . . . . . . . . . . . . . . . . . 515

Copper Patinas Are Also Beautiful . . . . . . . . . . . . . . . . . . . 515

Cathodic Protection . . . . . . . . . . . . . . . . . . . 517

Electrochemical Machining . . . . . . . . . . . . . . . . . . . 517

Metal Cleaning . . . . . . . . . . . . . . . . . . . . . 517

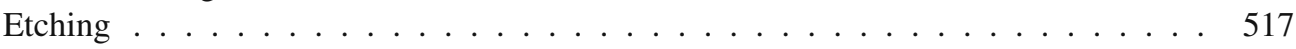

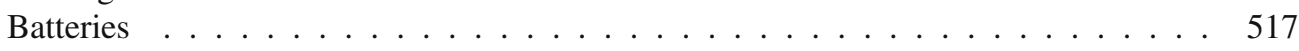

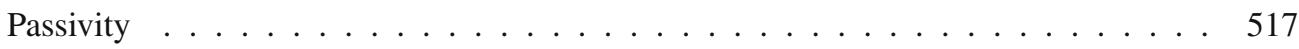

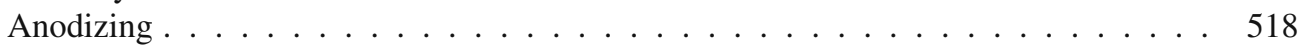

Titanium Jewelry and Art . . . . . . . . . . . . . . . . . . . 518

Caution to Inexperienced Artisans: . . . . . . . . . . . . . . . . . . . . . . 518

References . . . . . . . . . . . . . . . . . . . . 518

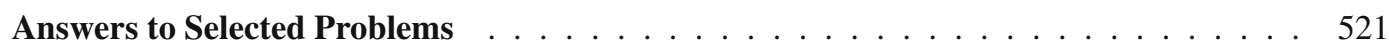

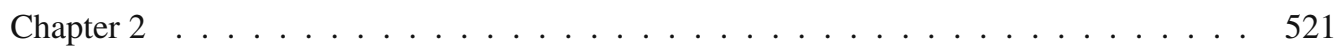

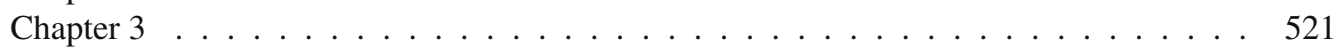

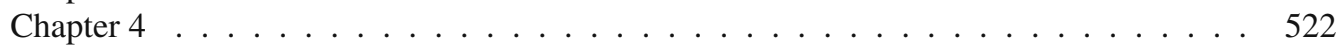

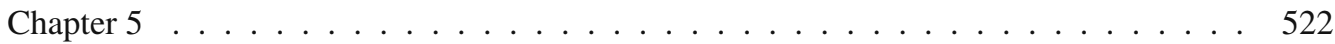

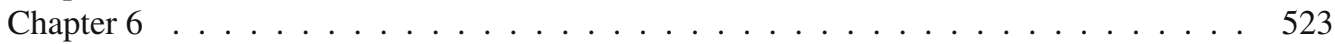

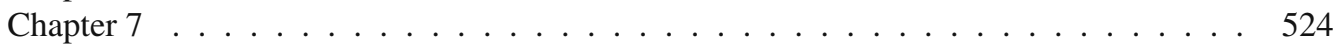

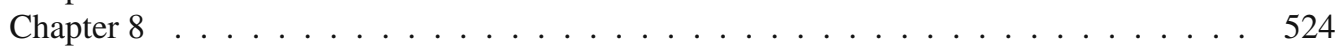

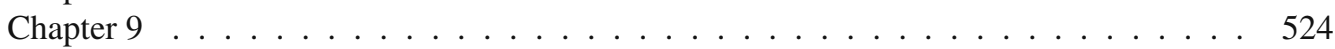

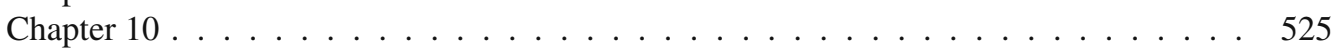

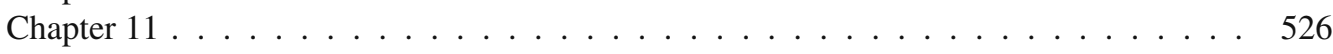

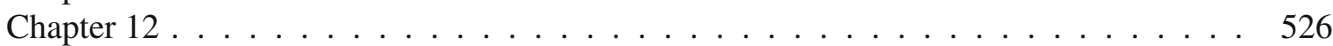




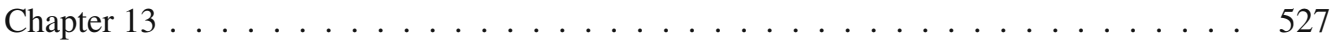

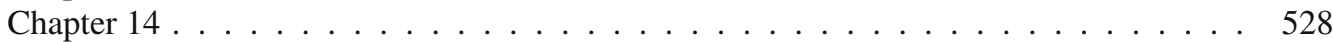

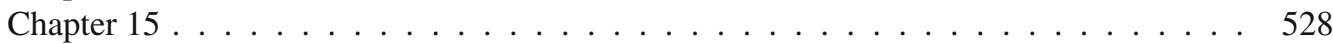

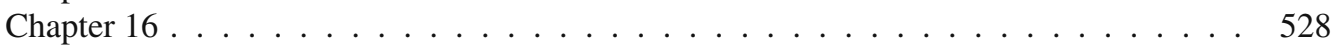

Appendix A: Some Properties of Various Elemental Metals . . . . . . . . . . . . . 531

Appendix B: Thermodynamic Relationships for Use in Constructing Pourbaix

Diagrams at High Temperatures $\ldots \ldots \ldots \ldots \ldots$

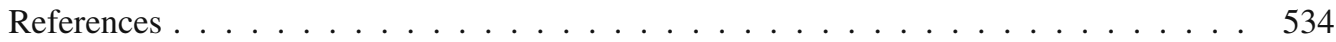

Appendix C: Relationship Between the Rate Constant and the Activation

Energy for a Chemical Reaction . . . . . . . . . . . . . . . . . . 535

Appendix D: Random Walks in Two Dimensions . . . . . . . . . . . . . . . . . . . . . 537

Appendix E: Uhlig's Explanation for the Flade Potential on Iron . . . . . . . . . . . . . 541

Appendix F: Calculation of the Randic Index $X(G)$ for the Passive Film

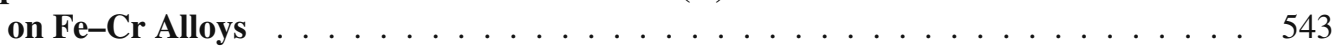

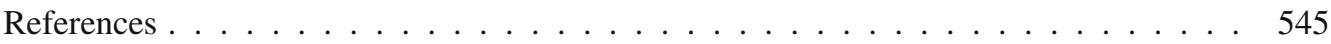

Appendix G: Acid Dissociation Constants $\mathbf{p} \boldsymbol{K}_{\mathbf{a}}$ of Bases and the Base Strength $\ldots \ldots 47$

Appendix H: The Langmuir Adsorption Isotherm ～. . . . . . . . . . . . . . . . . . 549

Appendix I: The Temkin Adsorption Isotherm ～. . . . . . . . . . . . . . . . . 551

Appendix J: The Temkin Adsorption Isotherm for a Charged Interface . . . . . . . . 553

Appendix K: Effect of Coating Thickness on the Transmission Rate of a Molecule Permeating Through a Free-Standing Organic Coating . . . . . . . . 557

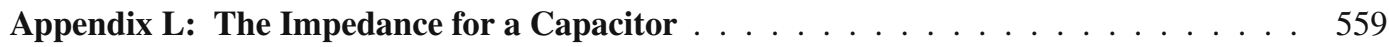

Reference ............................... 559

Appendix M: Use of L'Hospital's Rule to Evaluate $|\mathrm{Z}|$ for the Metal/Solution Interface for Large Values of Angular Frequency $\omega \ldots \ldots \ldots 1$

Appendix N: Derivation of the Arc Chord Equation for Cole-Cole plots . . . . . . . . 563 References . . . . . . . . . . . . . . . . . . . 565

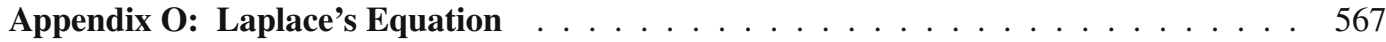

Reference . . . . . . . . . . . . . . . . . . . . 569

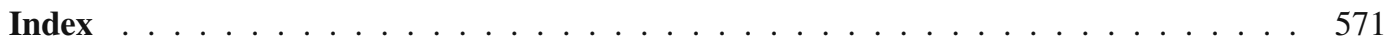

